\title{
Estimation of Radiation Dosimetry for some Common SPECT-CT Exams
}

\author{
Aida Mhiri ${ }^{1,}$, Ihsen Slim ${ }^{1}$, Mohamed Ghezaiel ${ }^{2}$ and Mohamed Faouzi Ben Slimène ${ }^{1}$ \\ ${ }^{1}$ Université de Tunis El Manar, Faculté de Médecine de Tunis, Salah AZAIEZ Institute, Nuclear Medicine \\ Department, 1007, Tunis, Tunisia \\ ${ }^{2}$ Université de Tunis El Manar, Faculté de Médecine de Tunis, Biophysic Department, 1007, Tunis, Tunisia
}

\begin{abstract}
Single photon emission computed tomography associated to $\mathrm{X}$ ray computed tomography (SPECT-CT) is a nuclear medicine tomographic imaging technique which improves diagnostic accuracy for particular clinical indications due to the possible attenuation and/or scatter correction of the SPECT functional images and the availability of helpful anatomic information. However, the introduction of CT in the nuclear diagnostic process results in a significant increase of the patient dose. Ideally is to establish sufficient image quality for a specific diagnostic task with the lowest effective dose to the patient in order to reduce stochastic effects. Indication of SPECT-CT should be made to adhere to the "As Low as Reasonably Achievable (ALARA)" principle and ensure that the patient is not subjected to unnecessarily high levels of radiation.

In this study, we evaluate effective doses received during some standard nuclear medicine exams from 100 patients who underwent SPECT-CT in our department and analyze parameters involved in variation of these doses according to the literature data.
\end{abstract}

Keywords: Single photon emission computed tomography (SPECT), X ray computed tomography (CT), Dosimetry, nuclear medicine.

\section{INTRODUCTION}

Single photon emission computed tomography associated to $\mathrm{X}$ ray computed tomography (SPECT$\mathrm{CT}$ ) is a nuclear medicine tomographic imaging technique which improves diagnostic accuracy for particular clinical indications due to the possible attenuation and/or scatter correction of the SPECT functional images and the availability of helpful anatomic information [1]. According to literature data SPECT-CT compared to SPECT alone causes more radiation to the patient which is not sufficient to cause deterministic effects. For SPECT effective dose depends on administered activity and patient's age. In fact the average radiopharmaceutical effective dose varies from varies from tens to thousands of $\mathrm{mSv}$ for some nuclear medicine exams [2]. However, the introduction of CT in nuclear diagnostic process results in a significant increase of the patient dose. In general, effective dose (E) for CT examinations can be higher than most other diagnostic imaging modalities [3]. Some authors have questioned the need to reduce doses particularly in children [4, 5]. A considerable choice of CT user-selectable exposure factors results in a significant variation in CT dose to the patient. In addition, in a nuclear medicine facility devoid of CT

*Address corresponding to this author at the Salah AZAIEZ Institute, Nuclear Medicine Department, Boulevard 9 Avril, Bab Saâdoun, 1006, Tunis, Tunisia;

Tel: + 21698356 038; E-mail: mhiri_aida@yahoo.fr technologists, there could potentially be limited knowledge of CT techniques, clinical applications, and associated dose consequences [6]. This study aims to evaluate effective doses received during some standard nuclear medicine exams with SPECT-CT and to analyze parameters involved in variation of these doses.

\section{METHODS}

Patient data from a dual-headed SPECT unit with an integrated 2-slice CT scanner (Symbia T E-Cam, SiemensMedical Systems, Erlangen, Germany) is presented in this study.

The CT parameters used were: tube current of 30 $90 \mathrm{mAs}$, slice thickness of $3-5 \mathrm{~mm}$, and tube voltage of 110-130 kV.

Data from 272 scans of common nuclear medicine procedures $\left({ }^{123} \mathrm{I}-\mathrm{MIBG},{ }^{99 \mathrm{~m}} \mathrm{Tc}-\mathrm{MIBI}\right.$ parathyroid, ${ }^{111} \mathrm{ln}$ octreotide, ${ }^{131} \mathrm{I}$ post therapy scan and ${ }^{99 \mathrm{~m}} \mathrm{Tc}-\mathrm{MDP}$ bone scan) comprising 100 patients between 20 and 83 years old (mean age: 56 years), were presented in this study.

For each patient the CT was acquired immediately after SPECT; the patient being kept in the same position to minimize offsets due to movement and allow proper registration on fused imaging. The contribution of total effective dose imparted by the nuclear tracers 
Table 1: Factors for DPL/Effective Dose Conversion Over Various Body Regions and Patient Ages [9]

\begin{tabular}{|c|c|c|c|c|c|}
\hline \multirow{2}{*}{ Region of body } & \multicolumn{5}{|c|}{ Effective dose per DLP $\left(\mathbf{m S v}(\mathbf{m G y ~} \mathbf{~ m})^{-1}\right)$ by ag } \\
\cline { 2 - 6 } & $\mathbf{0}$ year old & 1 year old & 5 year old & 10 year old & Adult \\
\hline \hline Head and neck & 0,013 & 0,0085 & 0,0057 & 0,0042 & 0,0031 \\
\hline Head & 0,011 & 0,0067 & 0,0040 & 0,0032 & 0,0021 \\
\hline Neck & 0,017 & 0,012 & 0,011 & 0,0079 & 0,0059 \\
\hline Chest & 0,039 & 0,026 & 0,018 & 0,013 & 0,014 \\
\hline Abdomen and pelvis & 0,049 & 0,030 & 0,020 & 0,015 & 0,015 \\
\hline Trunk & 0,044 & 0,028 & 0,019 & 0,014 & 0,015 \\
\hline
\end{tabular}

for each patient was calculated by multiplying the average administered activity for all patients by the "effective dose per unit administered activity" conversion factors listed in the International Commission on Radiological Protection (ICRP) Publication 53 [5] and $80[7,8]$.

The effective dose from the CT portion of the examination is estimated from the product of the dose length product (DLP) and a body-region-specific conversion factor, $k\left(\mathrm{mSv} \mathrm{mGy}^{-1} \mathrm{~cm}^{-1}\right)$, which take into account the varying biological sensitivities of different organs as given in Table 1 [9]. DLP is a patient-specific value determined by the scan length and the acquisition parameters; it represents the total amount of radiation delivered in the acquisition.

The CT scan was acquired immediately following completion of the SPECT study with the patient in the same position to minimize motion errors.

\section{RESULTS AND DISCUSSION}

The total effective dose received by each patient for SPECT-CT examination represents the sum of the effective dose induced by radiotracer administered to patient and effective dose induced by CT portion, according to the ICRP 80 recommendations [8]. Our results displayed on Tables 2 and 3 show that the average effective dose varies according to type of the exam: $1926,9 \mathrm{mSv}$ for ${ }^{131} \mathrm{I}$-post therapeutic scans, 5.4 $\mathrm{mSv}$ for ${ }^{123} \mathrm{I}$-MIBG scans and $4,2 \mathrm{mSv}$ for bone scans. The percentage increase of effective dose by the inclusion of the CT is about $125 \%$ for ${ }^{123}$-MIBG scans, $83 \%$ for bone scans, whereas it is negligible in case of treatment with ${ }^{131} \mathrm{I}(0.15 \%)$.

According to our results, and literature data [10,11], combined imaging results in a significant increase of the patient dose. It is true that the introduction of CT in the nuclear diagnostic process results in a significant

Table 2: The Average Effective Dose for the Total SPECT-CT Effective Dose for each Examination and the Percentage Increase in Effective Dose by the Inclusion of the CT

\begin{tabular}{|c|c|c|c|c|c|c|c|}
\hline \multirow[b]{2}{*}{$\begin{array}{l}\text { Type } \\
\text { of } \\
\text { study }\end{array}$} & \multirow[b]{2}{*}{$\begin{array}{c}\text { No } \\
\text { of } \\
\text { patients }\end{array}$} & \multicolumn{3}{|c|}{$\begin{array}{l}\text { Average Whole body effective dose in routine nuclear } \\
\text { medicine examinations }\end{array}$} & \multicolumn{2}{|c|}{$\begin{array}{c}\text { Average Effective dose for } \\
\text { the CT portion of the } \\
\text { routine nuclear medicine } \\
\text { examinations }\end{array}$} & \multirow{2}{*}{ 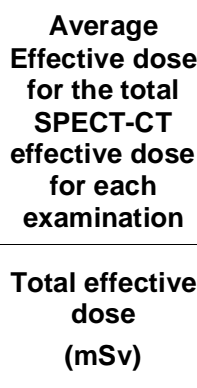 } \\
\hline & & $\begin{array}{l}\text { Average } \\
\text { Activity } \\
(\mathrm{MBq})\end{array}$ & $\begin{array}{l}\text { Dose per unit } \\
\text { activity } \\
\text { (mSv/MBq) }\end{array}$ & $\begin{array}{c}\text { Average } \\
\text { Effective dose } \\
(\mathrm{mSv})\end{array}$ & $\begin{array}{c}\text { Average } \\
\text { Effective dose } \\
(\mathrm{mSv})\end{array}$ & $\begin{array}{l}\text { Range } \\
\text { effective } \\
\text { dose } \\
(\mathrm{mSv})\end{array}$ & \\
\hline${ }^{99 \mathrm{~m}} \mathrm{Tc}-\mathrm{MDP}$ & 30 & 740 & 0.0057 & 4.2 & $3.5+/-1.2$ & $0.3-6.5$ & 7.7 \\
\hline $\begin{array}{l}{ }^{99 \mathrm{~m}} \mathrm{Tc}-\mathrm{MIBI} \\
\text { parathy }\end{array}$ & 20 & 925 & 0.009 & 8.3 & $2.3+/-0.7$ & $1.0-3.5$ & 10.6 \\
\hline $\begin{array}{l}{ }^{111} \text { In- } \\
\text { octreotide }\end{array}$ & 10 & 185 & 0.054 & 10 & $2.7+/-1.3$ & $1.9-5.4$ & 12.7 \\
\hline $\begin{array}{l}{ }^{131} \text { I post } \\
\text { therapy scan }\end{array}$ & 30 & 3700 & 0.52 & 1924 & $2.9+/-1.9$ & $1.2-5.9$ & 1926.9 \\
\hline${ }^{123}$ I-MIBG & 10 & 185 & 0.013 & 2.4 & $3.0+/-1.1$ & $1.4-5.8$ & 5.4 \\
\hline
\end{tabular}


Table 3: The Percentage Increase of Effective Dose by the Inclusion of the CT

\begin{tabular}{|c|c|c|}
\hline Type of Study & No of Patients & \% Increase of Effective dose by the inclusion of the CT \\
\hline \hline${ }^{99 m}$ Tc-MDP & 30 & $83 \%$ \\
\hline${ }^{99 m}$ Tc-MIBI parathyroid & 20 & $28 \%$ \\
\hline${ }^{111}$ In-octreotide & 10 & $27 \%$ \\
\hline${ }^{131}$ I post therapy scan & 30 & $0,15 \%$ \\
\hline${ }^{123}$ I-MIBG & 10 & $125 \%$ \\
\hline
\end{tabular}

increase of the dose delivered to patient, however, the radiation levels are well below the threshold for the occurrence of deterministic effects. Knowing that the main risk to patients is the occurrence of cancer and that this risk increases by $4 \%$ for every Sv of total effective dose received by a patient [9], in our series, this increased risk induced by additional exposure of the CT low dose, is insignificant and does not exceed $0.02 \%$. Taking into account the diagnostic contribution of a review SPECT- CT compared to SPECT examination alone, this "overhead" dose is justified because of the benefit to patient. However, this "additional" radiation should be optimized according to the desired objective (anatomical and / or attenuation correction) and CT parameters available on the machine. The most effective way to reduce doses received is to adapt irradiation protocols and acquisition parameters to the morphology of the patient [12].

For SPECT, the effective dose is dependent on administered activity and the patient's age. In CT, several methods are available to optimise and minimise the radiation absorbed doses [11, 13, 14]. Among the parameters which influence patient radiation dose and image quality: tube current, tube voltage, filtration, collimation, reconstruction method, reconstruction filter, slice thickness, pitch, and scanning length (Table 4) [12]. These parameters should be optimized for each specific examination and special efforts should be made with pediatric CT protocols [15].

The operator can monitor and modify most of them to obtain the necessary image quality with a minimal absorbed dose to the patient. Several studies have demonstrated an ability to affect radiation dose and image quality by using a lower tube voltage [16, 17]. The CT acquisition on the Symbia $T$ were performed using a tube current modulation system, Care Dose 4D (Siemens). CARE Dose 4D assesses the size of the patient cross section being scanned and adjusts tube current relative to the reference effective milliamperage. This system have a number of potential advantages, including better control of patient radiation dose, reduced load on the $x$-ray tube, and the maintenance of image quality [18].

Furthermore, patient dose must be as low as compatible with the medical purpose. Practice leading to a medical exposure must be clearly justified and protection against radiation must be optimized, particularly for children. Also, quality control procedures have to be defined because of the coupling between the two devices.

Finally, it should be noted that the reduction of patient exposure in CT is not only dependent on technological innovation, but also on the training of medical and paramedical staff. In fact, users are

Table 4: CT Parameters Influencing Radiation Exposure

\begin{tabular}{|c|}
\hline Radiation exposure from CT scan depends on operator dependent factors: \\
\hline \hline mAs: directly proportional to radiation dose \\
\hline KVp: not lienarry proportional to dose \\
\hline Pitch: inversly related to dose \\
\hline Slice thickness: requires an increase in mAs \\
\hline Number of scans: doubling the radiation dose \\
\hline Scanning mode: step- based vs spiral technique, single-slice vs multislice \\
\hline
\end{tabular}


required to undergo appropriate practical training to the purpose of radiological and / or scintigraphic practices to be aware and competent in radiation protection of the patient [19].

\section{CONCLUSION}

Despite the relative increase in radiation exposure associated with SPECT-CT is generally considered acceptable; reducing the patient dose should be a constant preoccupation of prescribing physician, nuclear physician's and qualified personnel performing the act. Indication of SPECT-CT must be clearly justified and protection against radiation must be optimized, particularly for children. Also, quality control procedures have to be defined because of the coupling between the two devices.

\section{REFERENCES}

[1] Mhiri A, Slim I, Ghezaiel M, Meddeb I, El Bez I, Yeddes I, et al. L'apport de l'imagerie hybride TEMP/TDM dans la prise en charge du cancer différencié de la thyrö̈de. Médecine Nucléaire. Imagerie Fonctionnelle et Métabolique 2012; 36: 554-60.

http://dx.doi.org/10.1016/j.mednuc.2012.08.008

[2] Larkin AM, Serulle Y, Wagner S, Noz ME, Friedman K. Quantifying the Increase in Radiation Exposure Associated with SPECT/CT Compared to SPECT Alone for Routine Nuclear Medicine Examinations. Int J Mol Imag 2011; 5 pages.

[3] Wall BF, Hart D, Revised radiation doses for typical X-ray examinations: report on a recent review of doses to patients from medical X-ray examinations in the UK by NRPB. Br J Radiol 1997; 70: 437-9.

[4] Valentin J. Managing patient dose in multi-detector computed tomography(MDCT) ICRP Publication 102. Annals ICRP 2007; 37: 1-79. DOI: 10.1016/j.icrp.2007.09.001

[5] The measurement, reporting and management of radiation dose. CT. Report of AAPM Task Group 23 no. 96, diagnostic Imaging Council CT Committee 2008.

[6] Radiation dose to patients from radiopharmaceuticals ICRP Publication 53. Annals ICRP 1987; 18: 1-4. http://www.icrp.org/docs/P053_addendum_4_\%28interim_20 02-10-15\%29.pdf

[7] Valentin J. Radiation dose to patients from radiopharmaceuticals (Addendum 2 to ICRP publication 53) ICRP publication 80 approved by the commission in September 1997. Annals ICRP 1998; 28: 1-126.
[8] International Commission on Radiological Protection. Radiation dose to patients from radiopharmaceuticals. Oxford, Pergamon Press 1987. Publication 53; 18: 4.

[9] Shrimpton PC, Hillier MC, Lewis MA, Dunn M. National survey of doses from CT in the UK. Br J Radiol 2003; 79 : 968-80. http://dx.doi.org/10.1259/bjr/93277434

[10] Larkin AM, Serulle Y, Wagner S, Noz ME, Friedman K. Quantifying the Increase in Radiation Exposure Associated with SPECT/CT Compared to SPECT Alone for Routine Nuclear Medicine Examinations. Int J Mol Imag 2011; Article ID 897202, doi:10.1155/2011/897202

[11] Mettler FA, Huda W, Yoshizumi TT, Mahesh M. Effective doses in radiology and diagnostic nuclear medicine: a catalog. Radiology 2008; 248: 254-63. doi: 10.1148/radiol.2481071451

[12] Office de Protection contre les Rayonnements Ionisants et Société Française de Radiologie. Les procédures radiologiques: Critères de qualité et optimisation des doses 2001.

[13] Kalender WA, Buchenau S, Deak P, Kellermeier M, Langner $\mathrm{O}$, et al. Technical approaches to the optimisation of CT. Physica Med 2008; 24: 71-9. http://dx.doi.org/10.1016/j.ejmp.2008.01.012

[14] Kalra MK, Maher MM, Toth TL, Hamberg LM, Blake MA, et al. Strategies for CT radiation dose optimization. Radiology 2004; 230: 619-28. http://dx.doi.org/10.1148/radiol.2303021726

[15] Mattsson S, Söderberg M. Radiation dose management in CT, SPECT/CT and PET/CT techniques. Radiat Prot Dosim 2011; 147: 13-21. http://dx.doi.org/10.1093/rpd/ncr261

[16] Frush DP. Pediatric dose reduction in computed tomography. Health Phys 2008; 95: 518-27.

\section{http://dx.doi.org/10.1097/01.HP.0000326335.34281.63}

[17] Funama Y, Awai K, Nakayama Y, Kakel K, Nagasue N, et al. Radiation dose reduction without degradation of low-contrast detectability at abdominal multisection CT with a low-tube voltage technique: phantom study. Radiology 2005; 237 905-10.

http://dx.doi.org/10.1148/radiol.2373041643

[18] Kalender WA, Deak P, Kellermeier M, van Straten M, Vollmar SV. Application- and patient size-dependent optimization of x-ray spectra for CT. Med Phys 2009; 36: 993-1007. http://dx.doi.org/10.1118/1.3075901

[19] Directive européenne 97/43/Euratom du Conseil du 30 ma 1996. Official J 1997; L 180: 22-7.

\section{DOI: http://dx.doi.org/10.6000/1927-3037.2012.01.04.7}

(C) 2012 Mhiri et al.; Licensee Lifescience Global.

This is an open access article licensed under the terms of the Creative Commons Attribution Non-Commercial License (http://creativecommons.org/licenses/by-nc/3.0/) which permits unrestricted, non-commercial use, distribution and reproduction in any medium, provided the work is properly cited. 